\title{
ORIGINAL
}

\section{PROTOCOLO DE VIGILANCIA SANITARIA DE TRABAJADORES CON PANTALLAS DE VISUALIZACIÓN DE DATOS: UNA VALORACIÓN DESDE LA PERSPECTIVA DE LA SALUD VISUAL (*)}

\author{
Ma del Mar Seguí Crespo (1), Elena Ronda Pérez (2), Alberto López Navarro (3), Pedro Vicente \\ Juan Pérez (4), Elena Tascón Bernabéu (5), Francisco Miguel Martínez Verdú (1).
}

(1) Departamento de Óptica, Farmacología y Anatomía. Universidad de Alicante.

(2) Área de Medicina Preventiva y Salud Pública. Universidad de Alicante.

(3) Servicio de Prevención. Clínica Optométrica. Universidad de Alicante.

(4) Servicio de Prevención. Unidad de Ergonomía y Psicosociología. Universidad de Alicante.

(5) Servicio de Prevención. Consellería de Justicia y Administraciones Públicas, Alicante.

\section{RESUMEN}

Fundamento: La vigilancia de la salud visual es esencial en la protección de trabajadores usuarios de pantallas de visualización de datos (PVD). En España, el protocolo más utilizado es el de Vigilancia Sanitaria Específica de PVD publicado en 1999 por el Ministerio de Sanidad y Consumo. El incremento de la producción científica sobre riesgos visuales ocupacionales asociados con ordenadores y la experiencia en su aplicabilidad durante esta última década justifican el objetivo de este trabajo: revisar la calidad del protocolo desde la perspectiva de la salud visual.

Métodos: Se utilizó una estrategia de consenso entre nueve expertos, mediante una técnica grupal mixta en dos fases consecutivas combinándose aspectos del método Delphi y del grupo nominal: evaluación individual del protocolo haciendo uso de la guía de consenso elaborada por los autores a partir del instrumento AGREE y posterior reunión para adoptar acuerdos y precisar las recomendaciones finales de mejora. Para el análisis se calculó la puntuación estandarizada de los bloques evaluados: alcance y objetivos, participación de los implicados, rigor en la elaboración, claridad y presentación, y aplicabilidad, así como la de las cuestiones. También se analizó la concordancia en las respuestas de los expertos.

Resultados: Todos los bloques obtuvieron puntuaciones por debajo del $50 \%$. La aplicabilidad, junto al rigor y la participación de los implicados durante la elaboración del protocolo, constituyeron los bloques más deficientes. Seis de los nueve expertos no recomendarían el protocolo y piensan que habría que reelaborarlo.

Conclusiones: El protocolo no alcanza la calidad adecuada para la vigilancia de la salud visual de los trabajadores usuarios de PVD. Los esfuerzos deben ir dirigidos a la mejora del mismo.

Palabras clave: Salud Laboral, Vigilancia de la Salud del Trabajador, Guía de Práctica Clínica, Terminales de Pantallas.

Correspondencia:

$\mathrm{M}^{\mathrm{a}}$ del Mar Seguí Crespo

Departamento de Óptica, Farmacología y Anatomía.

Universidad de Alicante

Carretera San Vicente del Raspeig s/n

San Vicente del Raspeig. 03690 Alicante.

Correo electrónico: MM.Segui@ua.es

\section{ABSTRACT \\ Health Surveillance Guide of Workers \\ Using Video Display Terminals: Evaluation from a Visual Health Perspective}

Background: Visual health surveillance is essential in the protection of workers who use video display terminals (VDT). In Spain, the most used is the Specific Health Surveillance Guide published in 1999 by the Ministry of Health. The increase of the scientific production upon computer related occupational visual hazards and the experience in its applicability during the last decade justify the aim of this work: reviewing the quality of the guide from the point of view of visual health.

Methods: A consensus strategy was used among nine experts by means of a mixed groupal technique in two consecutive stages combining some aspects of the Delphi method and of the nominal group: individual evaluation of the guide using the consensus guide made by the authors based on the AGREE instrument and the subsequent meeting in order to reach an agreement and to fix the final recommendations for improving it. For the analysis the standardized score of the review domains was calculated: scope and purpose, stakeholder involvement, rigour of development, clarity and presentation and applicability. It was also calculated for the items." Concordance in the answers of the experts was also analyzed.

Results: All domains obtained scores under $50 \%$. The applicability, rigour and stakeholder involvement during the development of the guide, where the most deficient domains. Six out of the nine experts would not recommend the guide and think it should be remade.

Conclusions: The guide does not reach the necessary quality for the surveillance of the visual health of the workers who use VDT. Efforts must be focussed to improve the guide.

Keywords: Occupational Health. Practice Guideline, Video Display Terminals.

(*) Trabajo financiado por el Instituto Nacional de Seguridad e Higiene en el Trabajo. Referencia: 606/UAL/PVDVIS 


\section{INTRODUCCIÓN}

Durante las últimas décadas la expansión de las nuevas tecnologías informáticas ha traído como consecuencia un incremento del uso de pantallas de visualización de datos (PVD) en todos los medios, incluido el medio laboral. El desarrollo de tareas que obligan al trabajador a permanecer de manera prolongada delante del ordenador se ha relacionado, entre otros efectos, con alteraciones oculares y visuales tales como la fatiga visual ${ }^{1,2}$. Una reciente revisión estima una prevalencia de esta dolencia del 90\% entre los 70 millones de trabajadores de los Estados Unidos que usan el ordenador más de tres horas al día ${ }^{3}$. En la VI Encuesta Nacional de Condiciones de Trabajo un $7,1 \%$ de los trabajadores manifiestan padecer fatiga visual derivada de su ocupación ${ }^{4}$.

La vigilancia de la salud visual es un elemento esencial en la protección de de los trabajadores expuestos a PVD. El Libro Blanco de la Vigilancia de la Salud ${ }^{5}$ señala que en España el Protocolo de Vigilancia Sanitaria Específica de Pantallas de Visualización de Datos editado por el Ministerio de Sanidad y Consumo (MSC) ${ }^{6}$ es el más utilizado por los profesionales de la prevención de riesgos laborales para el abordaje de este factor de riesgo. Este protocolo analiza los puestos de trabajo asociados al uso de PVD en cuanto a las condiciones de uso de las mismas y del entorno de trabajo, cuantificando el nivel de riesgo al que está sometido el trabajador y los efectos que comporta para su salud: alteraciones visuales, osteomusculares, cutáneas, influencia de las radiaciones en el embarazo y psicosomáticas, estableciendo las características específicas que debe reunir el examen de salud en este colectivo laboral ${ }^{6}$.

En nuestro país son todavía muy escasos los estudios de evaluación de los protocolos del MSC, pero en general han puesto de manifiesto como principal punto débil las dificultades para su aplicabilidad $^{7}$. Para que los protocolos cubran sus objetivos se debe comprobar la calidad de los mismos y asegurar que han sido rigurosos en minimizar sus potenciales $\operatorname{sesgos}^{8}$.

En salud visual, entendida como el enfoque dirigido a obtener una óptima eficacia funcional y fisiológica del sistema visual, la producción científica dedicada a tratar las alteraciones oculares o de percepción visual asociadas al uso de las nuevas tecnologías en el ámbito laboral ha ido en aumento en la última década ${ }^{1-3,9,10}$. Los conocimientos actuales consideran que la fatiga visual se puede desencadenar por causas distintas a la exposición a PVD ${ }^{10}$, lo que obliga a incluir en la vigilancia de la salud visual pruebas concretas ${ }^{11}$ para determinar si existen trabajadores con problemas refractivos ${ }^{12}$, acomodativos $o$ binoculares asociados que puedan alterar el efecto de la exposición ${ }^{13}$. Además, los profesionales demandan la adopción de instrumentos sensibles y específicos para garantizar la utilidad de los reconocimientos periódicos de los trabajadores ${ }^{14}$. Trascurridos diez años desde la fecha de su aprobación y con los últimos hallazgos sobre riesgos visuales ocupacionales en estos trabajadores, parece un momento adecuado para revisar el protocolo de PVD.

El objetivo de este trabajo es llevar a cabo una revisión de la calidad del Protocolo de Vigilancia Sanitaria Específica de Pantallas de Visualización de Datos del MSC, en aquellos aspectos relacionados con la vigilancia de la salud visual.

\section{SUJETOS Y METODOS}

Para llevar a cabo la evaluación del Protocolo de Vigilancia Sanitaria Específica de PVD, se estableció una estrategia de con- 
senso estructurado, mediante la utilización de una técnica grupal mixta en dos fases consecutivas, donde se combinaron aspectos característicos de la técnica Delphi y del grupo nominal o panel de expertos (figura 1). En concreto, participaron nueve profesionales (anexo 1) de disciplinas afines al tema de investigación: medicina del trabajo, epidemiología ocupacional, visión binocular y ergonomía; todos con experiencia en elaboración y/o aplicación de protocolos clínicos y de vigilancia de la salud.

Ante la falta de herramientas para la valoración de protocolos sanitarios bajo la perspectiva concreta de salud visual, el instrumento para la evaluación del protocolo consistió en una guía de consenso elaborada por los autores a partir de la versión española de la guía AGREE ${ }^{15}$, en la que se le introdujeron las modificaciones necesarias para que tuviera este enfoque. La guía AGREE $^{16}$ está validada y cuenta con el mayor grado de aceptación para la evaluación de guías de práctica clínica y protocolos sanitarios ${ }^{17-20}$. En España ya ha sido utilizada para la valoración del protocolo de asma laboral $^{7}$. Tras un diseño inicial de la guía de consenso, los autores llevaron a cabo un estudio piloto para evaluar el grado de comprensión de las preguntas, si la escala de respuesta era adecuada y si existía rechazo a contestar alguna de las preguntas. El estudio piloto conllevó la realización de algunos cambios menores en la formulación de las preguntas, hasta llegar a la versión definitiva de la guía de consenso que se presenta en el anexo 2 .

La guía de consenso incluye cuestiones que valoran la metodología utilizada en la elaboración del protocolo, sus contenidos y los factores relacionados con su aplicación. Consta de trece ítems organizados en cinco bloques: El primer bloque (ítems 1 y 2) analiza si los objetivos del protocolo se ajustan al problema de salud visual que se plantea y si hay una descripción clara de la población diana a la que va dirigido. El segundo blo- que (3 a 5) revisa el perfil de los profesionales que participaron en su elaboración, si se han considerado las experiencias de los trabajadores expuestos y si se ha realizado algún estudio piloto previo. El tercer bloque (6 a 9) valora si los contenidos están basados en la evidencia científica disponible y actualizada. El cuarto bloque (10 a 12) evalúa la claridad para especificar los exámenes visuales adecuados, quién los debe realizar, y cómo realizarlos. Por último, el quinto bloque (ítem 13) valora la aplicabilidad del protocolo. Para estos cinco bloques, la guía contempla una opción de respuesta cerrada mediante una escala de 4 puntos, que mide la intensidad de cumplimiento del protocolo ante una determinada cuestión. Si el protocolo la cumple por completo la respuesta debe ser igual a 4 (muy de acuerdo). Si en protocolo no cumple la cuestión planteada o si no hay información disponible al respecto, se opta por 1 (muy en desacuerdo). Si no es seguro que el protocolo cumple la cuestión por completo o si la información no es clara, según el grado, se escoge 2 (en desacuerdo) ó 3 (de acuerdo). La guía concluye con el ítem 14, que está destinado a una evaluación global del protocolo, con tres opciones de respuesta: sí lo recomendaría, lo recomendaría con condiciones o modificaciones, y no lo recomendaría, habría que reelaborarlo. Cada ítem se acompañó de una aclaración sobre la cuestión formulada y los autores podían incorporar un comentario justificando los motivos de su respuesta.

La guía de consenso se envió a los nueve expertos por correo electrónico, acompañada por una carta de presentación que precisaba los aspectos metodológicos a seguir. Los expertos efectuaron la evaluación, devolviendo la guía debidamente cumplimentada a los autores. Las contestaciones se organizaron agrupando los discursos comunes, con la finalidad de que quedara patente la opinión más refrendada. Estos resultados se remitieron de nuevo a los expertos. 
Figura 1

Fases del estudio para la evaluación y la mejora del Protocolo de Vigilancia Sanitaria Específica de Pantallas de Visualización de Datos
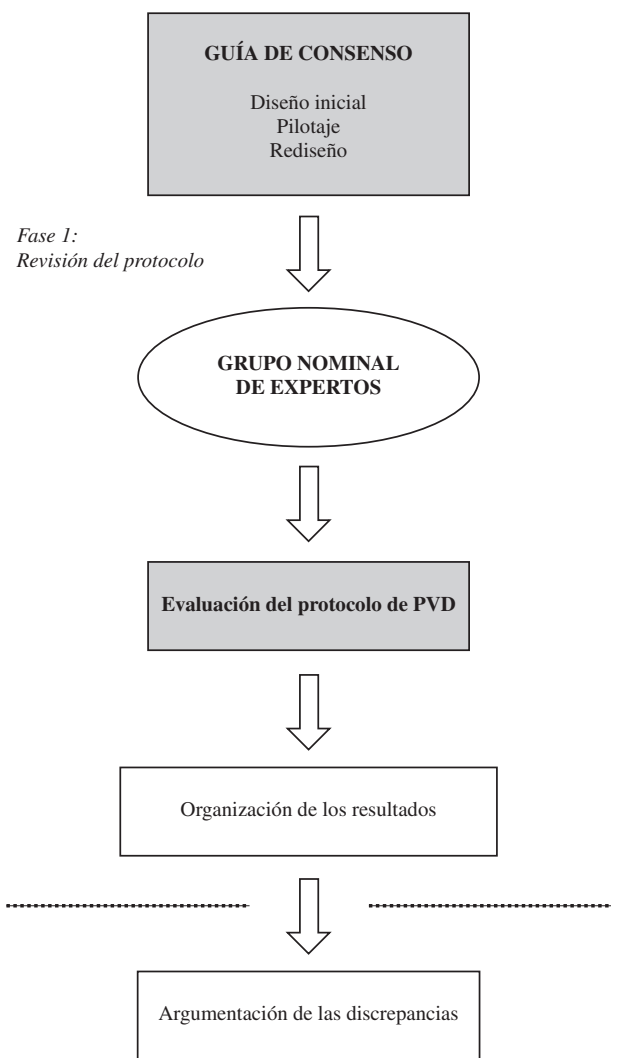

Fase 2:

Propuesta de mejora

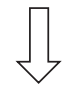

REUNIÓN

DE

CONSENSO

$\sqrt{5}$

Definición de las principales recomendaciones

Con esta información los expertos estaban en disposición de abordar la segunda fase del estudio. Cada uno por separado pudo preparar su argumentación ante aquellas de sus opiniones que habían sido divergentes con respecto al grupo. Esta fase culminó con una reunión del equipo investigador con el grupo de expertos que sirvió para consolidar los acuerdos obtenidos, y aproximar posturas en los aspectos donde inicialmente no había existido coincidencia. Todos los participantes aportaron sus ideas, debatiendo los aspectos más conflictivos. Las recomendaciones finales se extrajeron de los argumentos más aceptados por los asistentes y siempre con la intención de mejorar el protocolo.

La reunión estuvo debidamente documentada en base al conocimiento científico disponible. Se realizaron búsquedas bibliográficas en las bases de datos Medline, Cinahl, Scopus, Web of Knowledge y CISDOC, relacionadas con las alteraciones visuales asociadas al uso de pantallas de visualización de datos, y en concreto, de lo publicado sobre fatiga visual. También se consultaron páginas web de instituciones y entidades, en busca de otros protocolos de similares características.

Para el análisis cuantitativo de las respuestas cerradas, se calculó la puntuación estandarizada por bloque tal y como se establece en el instrumento AGREE:

- Puntuación obtenida = suma de puntuaciones otorgadas por las personas evaluadoras al bloque evaluado

- Mínima puntuación posible $=1$ punto (muy en desacuerdo) x 9 evaluadores $\times \mathrm{n}^{\circ}$ de ítems que tiene el bloque

- Máxima puntuación posible = 4 puntos (muy de acuerdo) x 9 evaluadores $\times n^{\circ}$ de ítems que tiene el bloque

La puntuación estandarizada será:

Puntuación obtenida - Mínima puntuación posible Máxima puntuación posible - Mínima puntuación posible $\times 100$ 
Igualmente se calculó la puntuación estandarizada para cada ítem, con el propósito de llegar a un mayor nivel de detalle en la identificación de los puntos fuertes y débiles del protocolo. Para la interpretación de las puntuaciones estandarizadas se utilizó la escala simétrica de seis rangos propuesta por Maqueda en $2006^{7}$.

Para el análisis de la concordancia se estableció el grado de concordancia, definido como la cantidad de participantes que habían optado por una misma opción de respuesta cerrada entre las cuatro alternativas posibles.

\section{RESULTADOS}

La tabla 1 presenta la puntuación obtenida en el análisis de los cinco bloques eva- luados. Se observa que ninguno de ellos alcanza una puntuación que supere el $50 \%$. Los dos bloques mejor valorados son el de alcance y objetivos y el de claridad y presentación que son clasificados como muy mejorables, con una puntuación estandarizada de $31,5 \%$ y $30,9 \%$ respectivamente. El bloque peor valorado corresponde al de aplicabilidad, clasificado muy deficiente con un $7,4 \%$.

De igual forma, en la puntuación obtenida independientemente en cada ítem (tabla 2), se aprecia que la calidad de todos ellos se sitúa por debajo del $50 \%$. Siete de los 13 ítems evaluados, presentan una calidad deficiente; muy deficiente en cuatro ítems y muy mejorable en tan sólo dos. En concreto, estos dos últimos corresponden a la valoración de si en el protocolo se describe específicamente

Tabla 1

Puntuación estandarizada obtenida en cada bloque y estimación de la calidad

\begin{tabular}{|l|c|c|}
\hline \multicolumn{1}{|c|}{ Bloque evaluado } & Puntuación estandarizada \% & \% Calidad* \\
\hline 1. Alcance y objetivos & 31,5 & Muy mejorable \\
4. Claridad y presentación & 30,9 & Deficiente \\
\hline 2. Participación de los implicados & 17,3 & Muy deficiente \\
3. Rigor en la elaboración & 13,0 & 7,4 \\
\hline 5. Aplicabilidad & & . \\
\hline
\end{tabular}

*Rangos de puntuación estandarizada \%: Excelente [90-100], Buena [70-90], Mejorable [50-70], Muy mejorable [30-50], Deficiente [10-30] y Muy deficiente $[0-10]^{7}$.

Tabla 2

Puntuación estandarizada obtenida en cada ítem y estimación de la calidad

\begin{tabular}{|c|c|c|}
\hline $\begin{array}{c}\text { Ítem evaluado } \\
\text { (Bloque al que pertenece) }\end{array}$ & $\begin{array}{c}\text { Puntuación estandarizada } \\
\%\end{array}$ & Calidad* \\
\hline $\begin{array}{l}12 \text { (Claridad y presentación) } \\
\mathbf{1} \text { (Alcance y objetivos) }\end{array}$ & $\begin{array}{l}44,4 \\
37,0\end{array}$ & Muy mejorable \\
\hline $\begin{array}{l}2 \text { (Alcance y objetivos) } \\
3 \text { (Participación de los implicados) } \\
4 \text { (Participación de los implicados) } \\
11 \text { (Claridad y presentación) } \\
\mathbf{7} \text { (Rigor en la elaboración) } \\
10 \text { (Claridad y presentación) } \\
\mathbf{6} \text { (Rigor en la elaboración) }\end{array}$ & $\begin{array}{l}25,9 \\
25,9 \\
25,9 \\
25,9 \\
22,2 \\
22,2 \\
18,5\end{array}$ & Deficiente \\
\hline $\begin{array}{l}9 \text { (Rigor en la elaboración) } \\
13 \text { (Aplicabilidad) } \\
8 \text { (Rigor en la elaboración) } \\
5 \text { (Participación de los implicados) }\end{array}$ & $\begin{array}{l}7,4 \\
7,4 \\
3,7 \\
0,0\end{array}$ & Muy deficiente \\
\hline
\end{tabular}

*Rangos de puntuación estandarizada \%: Excelente [90-100], Buena [70-90], Mejorable [50-70], Muy mejorable [30-50], Deficiente [10-30] y Muy deficiente $[0-10]^{7}$. 
algún objetivo con el propósito de prevenir alteraciones oculares y visuales (ítem 1) y de si los aspectos relativos a la salud visual se distinguen y clarifican de manera apropiada (ítem 12). La cuestión que valora si el protocolo ha sido probado en trabajadores expuestos susceptibles a sufrir alteraciones oculares y visuales (ítem 5) es la cuestión que ha obtenido peor puntuación estandarizada. En cuanto a los otros tres ítems valorados como muy deficientes, evalúan si el protocolo basa sus argumentos en la evidencia científica disponible (ítem 8), si establece la forma de actualizar sus contenidos de forma periódica (ítem 9) y si se discuten los costes potenciales que su aplicación implicaría para los servicios de prevención y las mutuas laborales (ítem 13).

La figura 2 representa el grado de concordancia entre los 9 expertos al contestar a cada uno de los ítems. La opción muy en desacuerdo es la respuesta más escogida

Figura 2

Grado de concordancia entre los nueve expertos para las cuatro opciones de respuesta en cada una de las preguntas

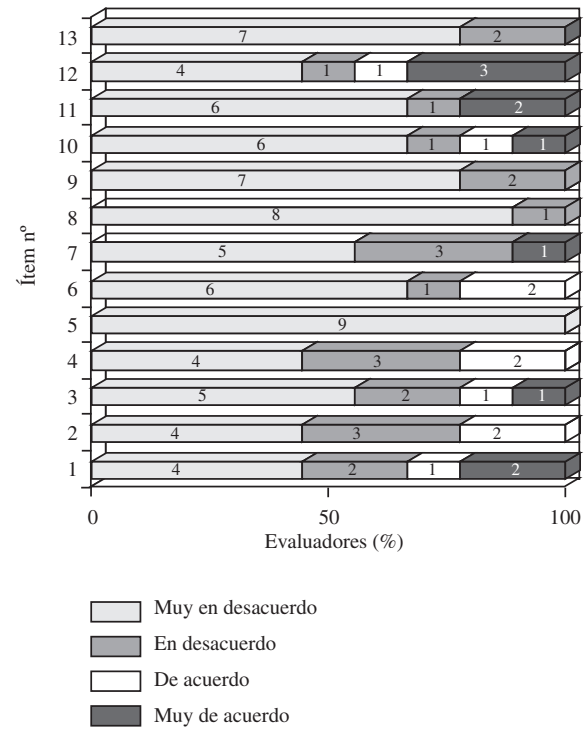

por los participantes en todos los ítems, presenta un grado de concordancia que supera el 50\% (más de la mitad del grupo de expertos coinciden en esta opción) en 9 de los 13 ítems. Esto señala una gran falta de cumplimiento del protocolo ante la mayoría de las cuestiones planteadas. Destacar que el ítem 5, ha conseguido unanimidad de opinión en torno al muy en desacuerdo, seguido de los ítems 8, 9 y 13 que presentan un grado de concordancia destacado en esta opción de respuesta. Por lo tanto, son los cuatro ítems con la calidad peor valorada (tabla 2), y además, esta opinión es compartida por gran parte de los expertos.

El grado de concordancia también permite conocer qué ítems fueron los más debatidos a lo largo de la reunión de consenso. Se debatieron con atención aquellas cuestiones en las cuales no aparece un grado de concordancia destacado en ninguna de las cuatro opciones de respuesta cerrada, en especial los ítems 1, 2, 4 y 12. De estas cuatro, destacar las cuestiones 1 y 12 que alcanzando la calidad más valorada (tabla 2), no contaban con un acuerdo previo entre participantes.

Finalmente, respecto a la evaluación global del protocolo, en la tabla 3 se observa que 6 expertos no recomendarían el protocolo para valorar las consecuencias de las PVD sobre la salud visual, y piensan que habría que reelaborarlo.

\section{Tabla 3}

Evaluación global del protocolo, respuesta al ítem 14 de la guía de consenso: ¿recomendaría este protocolo para valorar los efectos que sobre la salud ocular/visual de los trabajadores tienen las PVD?

\begin{tabular}{|l|c|}
\hline \multicolumn{1}{|c|}{ Respuesta } & $\begin{array}{c}\text { Número } \\
\text { de personas } \\
\text { evaluadoras }\end{array}$ \\
\hline Sí lo recomendaría & 1 \\
\hline Lo recomendaría con condiciones o modificaciones & 2 \\
\hline No lo recomendaría, habría que reelaborarlo & 6 \\
\hline
\end{tabular}




\section{DISCUSIÓN}

En este estudio se plantean una serie de recomendaciones con la intención de mejorar el protocolo de PVD desde la perspectiva de la salud visual, aportadas por nueve expertos, tras haber evaluado la calidad del mismo y haber obtenido deficientes resultados.

El bloque de alcance y objetivos es una de las partes que mejor puntuación ha obtenido. Aún así, se propuso la necesidad de incluir un objetivo específico de prevención de deterioros oculares y alteraciones visuales asociadas al uso de PVD que completará el de "establecer las características específicas que debe reunir el examen de salud en este colectivo laboral" que ya está contemplado en el protocolo. Respecto a las particularidades de la población trabajadora a la que va dirigido el protocolo, no se puede considerar de igual manera a todos los trabajadores expuestos; Stude$\mathrm{li}^{21}$ relacionó el aumento de la carga de trabajo con mayores molestias visuales, por ello en la vigilancia de la salud se debe tener en cuenta el tiempo frente al ordenador o la exigencia de la tarea ${ }^{22}$. Es decir, el protocolo debería definir la condición de trabajador usuario de PVD, incorporando los criterios de aplicación establecidos en la actual normativa de salud laboral ${ }^{23}$. Asimismo se deberían tener en cuenta aquellos trabajadores especialmente vulnerables a sufrir los efectos de la exposición. Tal es el caso de los trabajadores con determinadas ametropías ${ }^{24}$, présbitas ${ }^{25}$, usuarios de lentes de contacto ${ }^{26}$ o trabajadores con problemas de acomodación-convergencia $^{13}$.

En cuanto a la claridad y presentación del protocolo las recomendaciones están orientadas a paliar las deficiencias de los dos instrumentos que se incluyen para la vigilancia de la salud visual: el cuestionario de síntomas y el examen clínico. El cuestionario de síntomas debería ser un instrumento validado con un sistema de puntuación que permitiera definir criterios de normalidad. En este sentido se podría tener en cuenta los trabajos de Borsting ${ }^{27}$ y Rouse $^{28}$ sobre la validación de un cuestionario de síntomas oculares y visuales asociados a la insuficiencia de convergencia, que clasifica a los sujetos como sintomáticos para una sensibilidad de $97,8 \%$ y una especificidad de $87,0 \%$ según curvas ROC (Receiver Operating Characteristic), en las que el análisis del área bajo la curva es una medida de la exactitud de la prueba diagnóstica. Por otra parte, se considera fundamental adecuar el examen clínico a los estándares actuales ${ }^{29}$, concretando qué pruebas están al alcance del médico del trabajo y cuándo es necesario derivar a un especialista en salud visual para que realice los exámenes más específicos, pues se consigue reducir la fatiga visual si existe coordinación entre la medicina ocupacional y los especialistas en visión ${ }^{30}$. En todo caso, serán necesarios estudios que validen el Control Vision como herramienta para la vigilancia tal y como actualmente propone el protocolo, dado que recientemente Totaro $^{31}$ desaconsejaba el Ergovision Screener para la valoración visual en el entorno laboral frente a la convencional revisión de un especialista, e incluso se ha detectado un exceso de falsos positivos en las medidas con este tipo de instrumen$\operatorname{tos}^{32}$. Por lo que respecta a la presentación, se considera recomendable la inclusión de figuras de apoyo al texto, e incluso anexar un glosario de términos semejante al que incluye el protocolo francés de pantallas de visualización ${ }^{33}$.

$\mathrm{Al}$ evaluar la participación de los implicados, se identificó la ausencia de oftalmólogos y optometristas entre los componentes del grupo de trabajo que había elaborado el protocolo, lo que se considera una debilidad importante que podría subsanarse en futuras actualizaciones, en las que se demanda especificar al menos la especialidad de los autores. Además, se 
observó que el protocolo adolece de pilotaje previo y que las opiniones de los usuarios de PVD se incorporan de forma indirecta a través de los agentes sociales y de las sociedades científicas, sin que conste ninguna perteneciente al área de salud visual. Se propone habilitar procedimientos de participación directa, como cuestionarios o grupos de discusión entre trabajadores, médicos del trabajo y profesionales de la visión, para conocer las necesidades de los trabajadores expuestos.

En cuanto al rigor en la elaboración, no se identifica el proceso documental seguido para fundamentar sus contenidos, ni referencias en el texto. La bibliografía está compuesta exclusivamente de legislación y normas técnicas. Por ello se considera imprescindible, tras revisar los estudios científicos sobre salud visual ocupacional más relevantes de la última década, adecuar los contenidos del protocolo debidamente referenciados. Los expertos estimaron incluir un plazo de 5 años para futuras actualizaciones, salvo que los avances en la materia recomienden una renovación parcial previa anterior. Son muchos los estudios que incorporan criterios de evidencia científica a la elaboración de guías o procedimientos de vigilancia de la salud de los trabajadores, como única forma de paliar los problemas que se pueden plantear en los protocolos basados únicamente en el consenso ${ }^{34}$. Un ejemplo lo tenemos en la revisión de la evidencia efectuada por Waddell para el desarrollo de una guía para el control de la lumbalgia en el medio laboral ${ }^{35}$.

Por último, en relación a la aplicabilidad, los costes potenciales de su aplicación, no están descritos en ninguno de sus apartados. Convendría que el protocolo especificase cuáles son los recursos humanos y materiales para llevar a cabo una adecuada vigilancia de la salud visual de los trabajadores, incorporando los especialistas y nuevos equipos que se consideren necesarios.
En cuanto a las limitaciones del estudio, debemos tener en cuenta que la guía de consenso surge como una adaptación del instrumento AGREE. Al igual que éste, la guía de consenso se centra más en valorar el proceso de elaboración del protocolo que sus contenidos, siguiendo la creencia generalizada que asocia un buen proceso de elaboración con elevada calidad en los contenidos, lo cual no siempre es cierto ${ }^{18}$, ${ }^{20}$. Tampoco ha sido sometida a un riguroso proceso de validación, aunque ha habido un elevado grado de concordancia en las respuestas de los expertos. Con la elaboración de esta guía, se reconoce el interés expresado por determinados autores de disponer de adaptaciones del instrumento AGREE según requieran las especificidades de la vigilancia de la salud de los trabajadores $^{7}$.

Dado que la cuestión peor valorada del protocolo es la correspondiente a la no existencia de experiencia piloto alguna antes de que fuera editado por el MSC (ítem 5) y considerando el tiempo transcurrido desde su publicación, convendría hacer extensiva esta revisión a los servicios de prevención que cubren la vigilancia sanitaria de los sectores profesionales más asociados al uso de PVD. De esta manera se podrían detectar las deficiencias encontradas en la aplicación del mismo durante estos años. La consulta se podría efectuar a través de la propia guía de consenso, o bien mediante el envío de los resultados de este estudio para que los servicios de prevención puedan aportar ideas y completarlo.

En definitiva, la revisión del protocolo desde la perspectiva de la salud visual ha puesto de manifiesto que la mayor parte de las cuestiones evaluadas no alcanzan la calidad adecuada para llevar a cabo eficazmente la vigilancia de la salud visual de los trabajadores usuarios de PVD. El bajo nivel de recomendación global que obtiene el protocolo indica que los esfuerzos tie- 
nen que ir dirigidos a la mejora del mismo, fortaleciendo sus puntos más débiles. En este sentido se deberían considerar las propuestas aportadas por los expertos, dado que responden a un alto grado de consenso en la mayoría de las cuestiones evaluadas. No se debe olvidar que este protocolo constituyó un avance muy importante en la vigilancia de la salud, en cuanto a que supuso establecer las recomendaciones para orientar los reconocimientos a un factor de exposición incipiente en aquel momento, por ese motivo es importante actualizarlo adaptándolo a los conocimientos actuales y a la experiencia de estos diez años de aplicación. La incorporación de estas recomendaciones en futuras ediciones del protocolo, permitiría tomar decisiones razonadas acerca de la puesta en marcha de eficaces planes para la vigilancia de la salud visual de los trabajadores, especialmente si estos planes implican llevar a cabo costosas intervenciones ${ }^{36}$.

Este trabajo abre futuras líneas de trabajo, de aplicación del protocolo mejorado sobre una población de trabajadores expuestos a PVD.

\section{AGRADECIMIENTOS}

Este trabajo ha sido financiado por el Instituto Nacional de Seguridad e Higiene en el Trabajo (606/UAL/PVDVIS).

\section{BIBLIOGRAFÍA}

1. Woods V. Musculoskeletal disorders and visual strain in intensive data processing workers. Occup Med (Lond) 2005; 55(2): 121-7.

2. Tamez González S, Ortiz-Hernández L, MartínezAlcántara S, Méndez-Ramírez I. Riesgos y daños a la salud derivados del uso de videoterminal. Salud Publica Mex 2003; 45(3): 171-80.

3. Blehm C, Vishnu S, Khattak A, Mitra S, Yee RW. Computer Vision Syndrome: A Review. Surv Ophthalmol 2005; 50(3): 253-62.
4. Instituto Nacional de Seguridad e Higiene en el Trabajo. VI Encuesta Nacional de Condiciones de Trabajo. Madrid: Ministerio de Trabajo y Asuntos Sociales; 2007 [citado 16 de may. 2008]. Disponible en: http://www.mtas.es/insht/statistics/viencuesta.pdf

5. Junta de Extremadura. Consejería de Sanidad y Consumo. Libro blanco de la vigilancia de la salud para la prevención de riesgos laborales [citado 14 de mar. 2008]. Disponible en: http://www.aeemt.com/pdfs/LibroblancoV_Sal ud.pdf

6. Comisión de Salud Pública. Protocolos de Vigilancia Sanitaria Específica: Pantallas de Visualización de Datos. Madrid: Ministerio de Sanidad y Consumo; 1999 [citado 14 de mar. 2008]. Disponible en: http://www.msc.es/ciudadanos/saludAmbLaboral/docs/datos.pdf

7. Maqueda J, Buendía MJ, Gallego I, Muñoz C, Roel JM. Estudio de evaluación del Protocolo de Vigilancia Sanitaria Específica del Asma Laboral mediante el instrumento AGREE. Med Segur Trab 2006; 52(205): 13-21.

8. Soriano G. Protocolos para la Vigilancia de la Salud de los trabajadores: ¿dónde estamos? Arch Prev Riesgos Labor 2008; 11(2): 69-72.

9. Piccoli B. A critical appraisal of current knowledge and future directions of ergophthalmology: consensus document of the ICOH Committee on 'Work and Vision'. Ergonomics 2003; 46(4): 384-406.

10. Mocci F, Serra A, Corrias GA. Psychological factors and visual fatigue in working with video display terminals. Occup Environ Med 2001; 58(4): 267-71.

11. Jaschinski W. The proximity-fixation-disparity curve and the preferred viewing distance at a visual display as an indicator of near vision fatigue. Optom Vis Sci 2002; 79(3): 158-69.

12. Kotegawa Y, Hara N, Ono K, Arimoto A, Mukuno $\mathrm{K}$. Influence of accommodative response and visual symptoms on visual display terminal adult operators with asthenopia through adequately corrected refractive errors. Nippon Ganka Gakkai Zasshi 2008; 112(4): 376-81.

13. Hoffman DM, Girshick AR, Akeley K, Banks MS. Vergence-accommodation conflicts hinder visual performance and cause visual fatigue. J Vis 2008; 8(3): $33,1-30$.

14. Instituto Sindical de Trabajo Ambiente y Salud. II Foro ISTAS de Salud Laboral. Vigilar la salud, 
prevenir el riesgo [citado 14 de mar. 2008]. Disponible en: http://www.istas.ccoo.es/descargas/IIforo.pdf

15. The AGREE Collaboration. AGREE Instrument Spanish version [citado 23 de may. 2008]. Disponible en: http://www.easp.es/mbe/GPC-Tools/AGREE\% 20Espa\%C3\%B1ol\%202001.pdf

16. The AGREE Collaboration. Development and validation of an international appraisal instrument for assessing the quality of clinical practice guidelines: The AGREE project. Qual Saf Health Care 2003; 12(1): 18-23.

17. Rico Iturrioz R, Gutiérrez-Ibarlucea I, Asúa Batarrita J, Navarro Puerto MA, Reyes Domínguez A, Marín León et al. Valoración de escalas y criterios para la evaluación de Guías de Práctica Clínica. Rev Esp Salud Pública 2004; 78(4): 457-67.

18. Vlayen J, Aertgeerts B, Hannes K, Sermeus W, Ramaekers D. A systematic review of appraisal tools for clinical practice guidelines: multiple similarities and one common deficit. Int J Qual Health Care 2005; 17(3): 235-42.

19. Navarro Puerto MA, Ruiz Romero F, Reyes Domínguez A, Gutiérrez Ibarlucea I, Hermosilla Gago T, Alonso Ortiz del Río C et al. Are the guidelines guiding us reliable? Evaluation of the Spanish clinical practice guidelines. Rev Clin Esp 2005; 205(11): 533-40.

20. Cates JR, Young DN, Bowerman DS, Porter RC. An independent AGREE evaluation of the Occupational Medicine Practice Guidelines. Spine J 2006; 6(1): 72-7.

21. Studeli T, Menozzi M. Effect of subjetive and objetive workload on asthenopia at VDU workplaces. Int J Occup Saf Ergon 2003; 9(4): 441-51.

22. Travers PH, Stanton BA. Office workers and video display terminals: physical, psychological and ergonomic factors. AAOHN J 2002; 50(11): 489-93.

23. Instituto Nacional de Seguridad e Higiene en el Trabajo. Guía técnica para la evaluación y prevención de los riesgos relativos a la utilización de equipos con pantallas de visualización [citado 14 de mar. 2008]. Disponible en: http://www.mtas.es/insht/practice/pantallas.pdf

24. Tatemichi M, Nakano T, Tanaka K, Hayashi T, Nawa T, Miyamoto $\mathrm{T}$ et al. Possible association between heavy computer users and glaucomatous visual field abnormalities: a cross sectional study in Japanese workers. J Epidemiol Community Health 2004; 58: 1021-7.
25. Fenga C, Di Pietro R, Fenga P, Di Nola C, Spine1la R, Cacciola A et al. Asthenopia in VDT users: our experience. G Ital Med Lav Ergon 2007; 29(3 Suppl): 500-1.

26. González-Méijome JM, Parafita MA, YebraPimentel E, Almeida JB. Symptoms in a population of contact lens and noncontact lens wearers under different environmental conditions. Optom Vis Sci 2007; 84(4): 296-302.

27. Borsting EJ, Rouse MW, Mitchell GL, Scheiman M, Cotter SA, Cooper J et al. Validity and reliability of the revised convergence insufficiency symptom survey in children aged 9 to 18 years. Optom Vis Sci 2003; 80(12): 832-8.

28. Rouse MW, Borsting EJ, Mitchell GL, Scheiman M, Cotter SA, Cooper J et al. Validity and reliability of the revised convergente insufficiency symptom survey in adults. Ophthal Physiol Opt 2004; 24(5): 384-90.

29. Scheiman M, Wick B. Clinical management of binocular vision: heterophoric, accommodative, and eye movement disorders. $3^{\mathrm{a}}$ ed. Philadelphia: Lippincott Williams \& Wilkins, 2008.

30. Speeg-Schatz C, Hansmaennel G, Gottenkiene S, Tondre M. Travail sur écran et fatigue visuelle et son évolution après prise en charge ophtalmologique. J Fr Ophtalmol 2001; 24(10): 1045-52.

31. Totaro B, Assini R, Consonni D, Guzzi C, Troiano P, Dal Pozzo R et al. Adecuacy and reliability of orthoanalyzer Ergovision for job-fitness evaluation. G Ital Med Lav Ergon 2007; 29(3 Suppl): 250-1.

32. Horberry TJ, Gale AG, Taylor SP. Vision screeners for display screen equipment users: An experimental evaluation. Displays 1997; 17: 111-7.

33. L'Institut national de recherché et de sécurité. Écrans de visualisation. Santé et ergonomie [citado 14 de mar. 2008]. Disponible en: http://www.inrs.fr/INRSPUB/inrs01.nsf/inrs01_catalog_view_view/4CF47B8 1F1C8EAB8C1257060002991A5/\$FILE/ed924.pdf

34. Birrell L, Beach J. Developing evidence-based guidelines in occupational health. Occup Med (Lond) 2001; 51(2): 73-4.

35. Waddell G, Burton AK. Occupational health guidelines for the management of low back pain at work: evidence review. Occup Med 2001; 51(2): 124-35.

36. Cole BL. Do video display units cause visual problems? - a bedside story about the processes of public health decision-making. Clin Exp Optom 2003; 86(4): 205-20. 


\section{Anexo 1}

Relación de personas expertas que han participado en la evaluación del Protocolo de Vigilancia Sanitaria Específico de Pantallas de Visualización de Datos

\begin{tabular}{|l|l|}
\hline Chipont Benabent, Enrique & Clínica Oftalmológica OFTÁLICA, Alicante \\
\hline García Gómez, Montserrat & Subdirección General de Salud Ambiental y Salud Laboral, Ministerio de Sanidad y Consumo, Madrid \\
\hline González Díaz-Obregón, Enrique & Departamento de Óptica II (Optometría), Universidad Complutense de Madrid \\
\hline Lara Lacárcel, Francisco & Departamento de Oftalmología, Anatomía Patológica y Otorrinolaringología, Universidad de Murcia \\
\hline Lillo Jover, Julio & Departamento de Psicología Diferencial y del Trabajo, Universidad Complutense de Madrid \\
\hline Maqueda Blasco, Jerónimo & Escuela Nacional de Medicina del Trabajo, Instituto de Salud Carlos III, Madrid \\
\hline Parets Llorca, Rosario & Servicio de Prevención del INSS y Escuela de Medicina del Trabajo, Alicante \\
\hline Pedraza Poveda, Adriana Mutua & Mutua UNIVERSAL / FREMAP, Alicante \\
\hline Roel Valdés, José M & Instituto Sindical de Trabajo, Ambiente y Salud (ISTAS-CCOO), Alicante \\
\hline
\end{tabular}

Anexo 2

Estructura y contenido de la guía de consenso utilizada para la evaluación del Protocolo de Vigilancia Sanitaria Específica de Pantallas de Visualización de Datos desde la perspectiva de la salud visual

BLOQUE 1

Alcance y objetivos

1. El protocolo describe objetivos específicos para prevenir los deterioros oculares o las alteraciones visuales asociadas al uso de PVD.

2. Considera las particularidades visuales de los trabajadores expuestos en cuanto a sus características clínicas y condiciones de trabajo.

BLOQUE 2

Participación de los implicados

3. En su elaboración han participado especialistas en salud ocular y visión junto con especialistas en salud laboral

4. Para su elaboración se han considerado las necesidades de los sectores profesionales más vulnerables a sufrir alteraciones visuales.

5. Ha sido probado en trabajadores expuestos a PVD.

BLOQUE 3

Rigor en la elaboración

6. Explica la estrategia de búsqueda de la evidencia científica seguida en la elaboración del protocolo, relativa a las alteraciones oculares y visuales asociadas al uso de PVD.

7. Describe los criterios seguidos en la selección de contenidos relacionados con el sistema visual.

8. Las recomendaciones sobre salud visual están debidamente referenciadas.

9. Establece la forma de actualizar sus contenidos en materia de salud visual.

BLOQUE 4

Claridad y presentación

10. Establece diferentes opciones para el manejo de las alteraciones relacionadas con el sistema visual

11. El procedimiento a seguir para la vigilancia de la salud visual se explica sin ambigüedades.

12. Los aspectos oculares y visuales clave se presentan de una forma comprensible.

BLOQUE 5

Aplicabilidad

13. Se trata sobre los costes potenciales de su aplicación.

EVALUACIÓN GLOBAL:

14. ¿Recomendaría este protocolo para valorar los efectos que sobre la salud ocular/visual de los trabajadores tienen las PVD? 
